PREPARED FOR THE U.S. DEPARTMENT OF ENERGY, UNDER CONTRACT DE-AC02-76CH03073

PPPL-3824

PPPL-3824

UC-70

Noise Suppression and Enhanced Focusability in Plasma Raman Amplifier with Multi-frequency Pump

by

A.A. Balakin, G.M. Fraiman, N.J. Fisch, and V.M. Malkin

June 2003

N/M

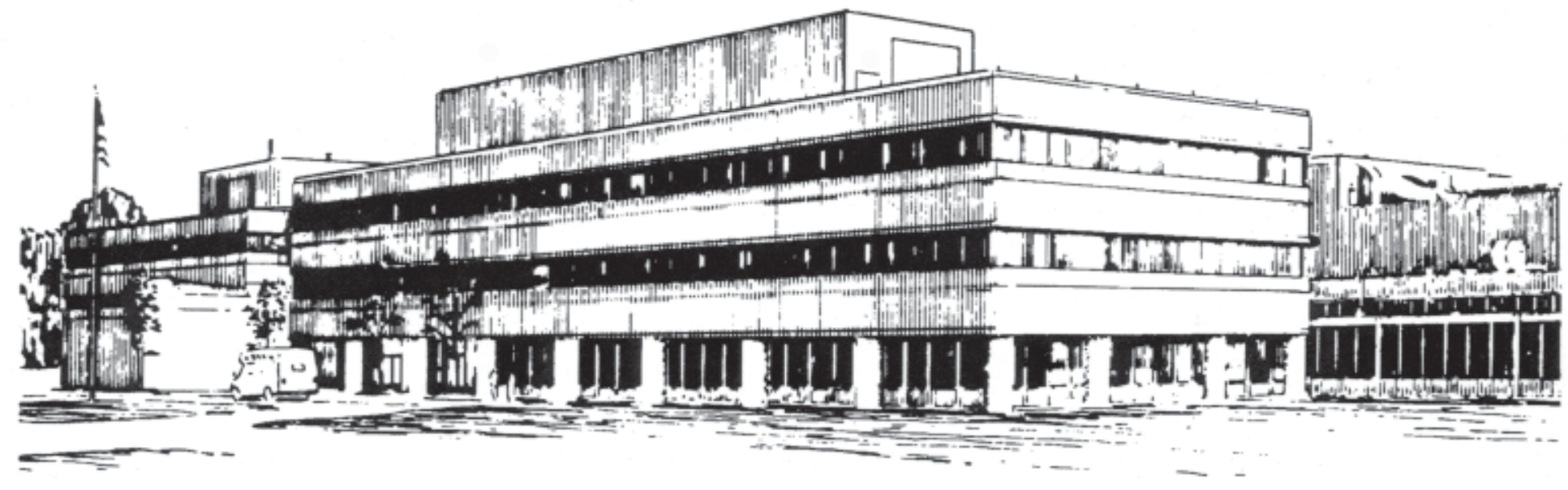

PRINCETON PLASMA PHYSICS LABORATORY PRINCETON UNIVERSITY, PRINCETON, NEW JERSEY 


\section{PPPL Reports Disclaimer}

This report was prepared as an account of work sponsored by an agency of the United States Government. Neither the United States Government nor any agency thereof, nor any of their employees, makes any warranty, express or implied, or assumes any legal liability or responsibility for the accuracy, completeness, or usefulness of any information, apparatus, product, or process disclosed, or represents that its use would not infringe privately owned rights. Reference herein to any specific commercial product, process, or service by trade name, trademark, manufacturer, or otherwise, does not necessarily constitute or imply its endorsement, recommendation, or favoring by the United States Government or any agency thereof. The views and opinions of authors expressed herein do not necessarily state or reflect those of the United States Government or any agency thereof.

\section{Availability}

This report is posted on the U.S. Department of Energy's Princeton Plasma Physics Laboratory Publications and Reports web site in Fiscal Year 2003. The home page for PPPL Reports and Publications is: http://www.pppl.gov/pub_report/

DOE and DOE Contractors can obtain copies of this report from:

U.S. Department of Energy

Office of Scientific and Technical Information

DOE Technical Information Services (DTIS)

P.O. Box 62

Oak Ridge, TN 37831

Telephone: (865) 576-8401

Fax: (865) 576-5728

Email: reports@adonis.osti.gov

This report is available to the general public from:

National Technical Information Service

U.S. Department of Commerce

5285 Port Royal Road

Springfield, VA 22161

Telephone: $1-800-553-6847$ or

(703) $605-6000$

Fax: (703) 321-8547

Internet: http://www.ntis.gov/ordering.htm 


\title{
Noise suppression and enhanced focusability in plasma Raman amplifier with multi-frequency pump
}

\author{
A.A. Balakin ${ }^{a}$, G.M. Fraiman ${ }^{a}$, N.J. Fisch ${ }^{b, c}$, V.M. Malkin ${ }^{c}$ \\ a Institute of Applied Physics RAS, Nizhnii Novgorod, Russia 603950 \\ ${ }^{b}$ Princeton Plasma Physics Laboratory, Princeton, NJ USA 08543 \\ ${ }^{c}$ Department of Astrophysical science, Princeton University, Princeton, NJ USA 08543
}

\begin{abstract}
Laser pulse compression/amplification through Raman backscattering in plasmas can be facilitated by using multi-frequency pump laser beams. The efficiency of amplification is increased by suppressing the Raman instability of thermal fluctuations and seed precursors. Also the focusability of the amplified radiation is enhanced due to the suppression of large-scale longitudinal speckles in the pump wave structure.
\end{abstract}

\section{INTRODUCTION}

The amplification by resonant Raman backscattering in plasmas currently represents one of the most promising ways of generating ultra-intense short laser pulses. Compared to the conventional technique of chirped-pulse amplification, in principle unfocused output intensity can be $10^{4}-10^{5}$ times higher. In achieving these amplification effects, two optical systems may be utilized: one system for handling high power and fluence, and a second focusing system handling only low power. Since the focusing system does not operate near damage threshold limits nor is subject to thermal stresses, the focusing optics can be optimized for precise focusing [1]Currently, a number of difficulties exist on the way to the experimental realization of a plasma Raman amplifier. One is propagating an intense laser pump through plasma to the region of interaction with the amplified pulse. For the most promising regimes of amplification, a pump intensity is needed on the order of $10^{14}-10^{16} \mathrm{~W} / \mathrm{cm}^{2}$ with pulse duration of a fraction of a nanosecond [2]. If the plasma cross-section is several centimeters, then tremendous power should be focused in target. For such application however, it is difficult to imagine such an energetic laser pulse using a single laser. However, using an intense pump comprising several laser beams with equal frequencies may not be the optimal solution.

One can imagine a number of difficulties in using multiple pumps. If the pump is formed by multiple but equivalent laser beams, large-scale longitudinal speckles appear in the pump structure. This results in the appearance of a small-scale transverse modulation of the amplified pulse and thus leads to poor focusability of the latter. Also, as an intense pump with a fixed frequency passes through plasma, thermal Langmuir fluctuations $[2,3]$ and seed precursors are amplified by the same Raman mechanism being used for the desired signal amplification [4]. The precursor can absorb a significant part of the pump energy. The pump depletion leads to lower efficiency of the desired pulse amplification and, finally, to lower intensity of the output radiation. Similar problems have been addressed within the context of inertial confinement fusion, where amplification of fluctuations was avoided through various laser beam smoothing tech- niques [5]. However, in the case at hand, where backscatter from thermal fluctuations is to be avoided at the same time that backscatter of the desired seed pulse proceeds, the usual methods of noise suppression do not apply.

In ideally uniform plasmas, the Raman instability of the plasma noise and precursor amplification is suppressed by chirping the pump wave $[2,3]$. The idea of using a chirped pump can be briefly put as follows. Linear amplification of each spectral component of plasma noise is limited by detuning $\delta \omega$ from the three-wave resonance between the two electromagnetic pulses and the Langmuir wave. Providing that $\delta \omega$ is changing in time because of linear variation of the pump frequency $\omega_{0}$, each component of the plasma thermal fluctuations spectrum can be amplified only by a finite factor. If that is small enough (meaning that $\omega_{0}$ is changing sufficiently fast), thermal fluctuations, though amplified, remain in the linear regime and do not deplete the pump wave significantly. On the other hand, nonlinear amplification of the desired signal persists because of the spectral broadening of the latter, as the desired signal is being compressed during the interaction with the pump.

Though efficient for uniform plasmas, pump chirping itself may not provide focusability of the amplified pulse if quasi-static density perturbations are present in plasma. Effectively, density perturbations $\delta n$ can be considered as additional chirping $\delta \omega \propto \partial(\delta n) / \partial z$, which, since random in the transverse direction, leads to random distortion of the amplified pulse phase front. As shown in Ref. [6], already for $\delta n / n \sim 3 \%$ (where $n$ is the mean electron density) with correlation length $l_{\text {corr }}=300 \mu \mathrm{m}$, the desired signal becomes practically unfocusable after amplification. As $l_{\text {corr }}$ grows, the maximal possible amplitude of density perturbations allowing decent focusability decreases as $1 / \sqrt{l_{\text {corr }}}[6]$.

However, there exists an even more strict limitation on the amplitude of quasi-static density perturbations. Even with pump chirping, the presence of those density inhomogeneous results in the development of an instability of thermal Langmuir fluctuations [7]. As follows from Ref. [7, 8], this noise amplification can be considered as a parametric instability, most dangerous for $l_{\text {corr }} \gamma / c \lesssim 2$, where $\gamma=a_{0} \sqrt{\omega_{0} \omega_{p} / 2}$ is the linear growth rate of the Raman instability [2], $c$ is the speed of light, 
$a_{0}, \omega_{0}$ are the amplitude and frequency of the pump and $\omega_{p}$ is the plasma frequency. Already at $\delta n / n=0.3 \%$, noise suppression by means of linear chirping (that is, when $\delta \omega$ is changing linearly on time) becomes inefficient for $l_{\text {corr }} \gamma / c \leq 1$. In the parameter region of interest (pump intensity $10^{14} \mathrm{~W} / \mathrm{cm}^{2}$, pump wavelength $\lambda=1 \mu \mathrm{m}, n=7 \times 10^{18} \mathrm{~cm}^{-3}$ ), this corresponds to $l_{\text {corr }} \leq 130 \mu \mathrm{m}$.

The paper is organized as follows. In Sec. II we describe the equations being simulated and the used numerical code. In Sec. III we introduce the multiple beams pump (MBP) scheme and discuss its features. In Sec. IV we show how the MBP scheme can be used to enhance the focusability of the seed. In Sec. V we show the noise suppression in plasma with density fluctuations using the MBP scheme. In Sec. VI we show how the idea of MBP can be modified for a single beam pump. In Sec. VIII we suggest a number of practical recommendations for using the MBP and offer conclusions. sinusoidally chirped pump.

\section{BASIC EQUATIONS AND CODE OUTLINE}

Below, we present the results of numerical simulations of the seed pulse Raman amplification. The numerical scheme involved solving the two-dimensional equations describing the three-wave interaction process [2]:

$$
\begin{aligned}
& \partial_{t} a+\partial_{z} a-i \nabla_{\perp}^{2} a=b f, \\
& \partial_{t} b-\partial_{z} b-i \nabla_{\perp}^{2} b=-a f^{*}, \\
& \partial_{t} f+i \delta \omega f=-a b^{*}+S-\nu f,
\end{aligned}
$$

where $a$ and $b$ are the amplitudes of the vector potentials of the pump wave and the amplified pulse respectively measured in units $m_{e} c \omega_{0} / e, f$ is the amplitude of the plasma wave electric potential measured in units $\left(m_{e} c \omega_{0} / 2 e\right) \sqrt{\omega_{p} / 2 \omega_{0}}, S$ is the thermal plasma fluctuations source, the pump frequency $\omega_{0}$ is much larger than the plasma frequency $\omega_{p}=\sqrt{4 \pi n e^{2} / m_{e}}$ and can be considered in all these coefficients equal to the amplified pulse frequency, $e$ and $m$ are the electron charge and mass respectively; the time $t$ is measured in units $t_{0}=\sqrt{2 / \omega_{0} \omega_{p}}$, the longitudinal coordinate $z$ is measured in units $c t_{0}$, the detuning $\delta \omega$ is measured in units $t_{0}^{-1}$, and $\nu$ is the Langmuir wave damping including collisional and Landau damping.

In the calculations presented below the pump wave amplitude is $a_{0}=0.006$ with Gaussian transverse profile with width $10 \mathrm{~cm}$, the frequency of pump is $\omega_{0}=$ $2 \times 10^{15} \mathrm{sec}^{-1}$. For such parameters, the linear e-folding length, calculated according to the linear growth rate of monochromatic pump backscattering instability $\gamma=$ $a_{0} \sqrt{\omega_{0} \omega_{p} / 2}$, is $c / \gamma=130 \mu \mathrm{m}$. We used the length of plasma $7 \mathrm{~mm}$ or $55 \mathrm{c} / \gamma$. Note that the plasma length is much smaller than difraction length $d \approx 10^{5} \mathrm{~cm}$ for these parameters. As result the effect of diffraction is not essential in further simulations. The initial seed pulse had the same transverse Gaussian profile as the pump, but the twice smaller amplitude and duration $40 \mathrm{fs}$ (length is $12 \mu \mathrm{m})$ :

$$
b_{0}=\frac{a_{0}}{2} e^{-(t / 40 \mathrm{fs})^{2} / 2} F(r), \quad F(r)=e^{-(r / 10 \mathrm{~cm})^{2} / 2} .
$$

Numerical simulation was performed by a new very fast hydrodynamic code "MBRS" which was created specifically for BRA simulation. This code allows to include into consideration a wide spectrum of effects. There are 3 -wave interaction, nonlinearity of EM waves, plasma thermal noises, plasma density fluctuations, plasma wave dumping and wave-breaking, transverse broadening due to diffraction and others effects are included in the code at current time. The structure of the code is such that any hydrodynamic-like effects can be easily included into consideration. The code uses exact solution of the part of equations (like method of "exponent operator" [9]) to maximize the internal calculation step and reduce the numerical errors. The code is fully parallelized for using in computer cluster so that the calculation time of typical $2 \mathrm{D}$ variant with grid size $128 \times 2750$ pointsis 10 minutes or less on 15 processes AMD Athlon $1.7 \mathrm{GHz}$. Analogous $3 \mathrm{D}$ simulation with grid size $64 \times 64 \times 2750$ points takes 8 hours on equal cluster. The code was tested with program described in [10].

\section{MULTIPLE BEAMS PUMP}

We propose a method of efficient compression and focusing in the presence of thermal plasma fluctuations and plasma density fluctuations of arbitrary correlation length $l_{\text {corr }}$. The proposed method leads also stabilizes the seed precursors and preserves good focusability of the amplified pulse. The method consists of using a pump wave formed by multiple laser beams of slightly different frequencies $\omega_{0}+i \Delta \omega_{n}\left(\Delta \omega_{n} \ll \omega_{0}\right)$ and wavevectors $\boldsymbol{k}_{0}+\Delta \boldsymbol{k}_{n}$ (Fig. 1):

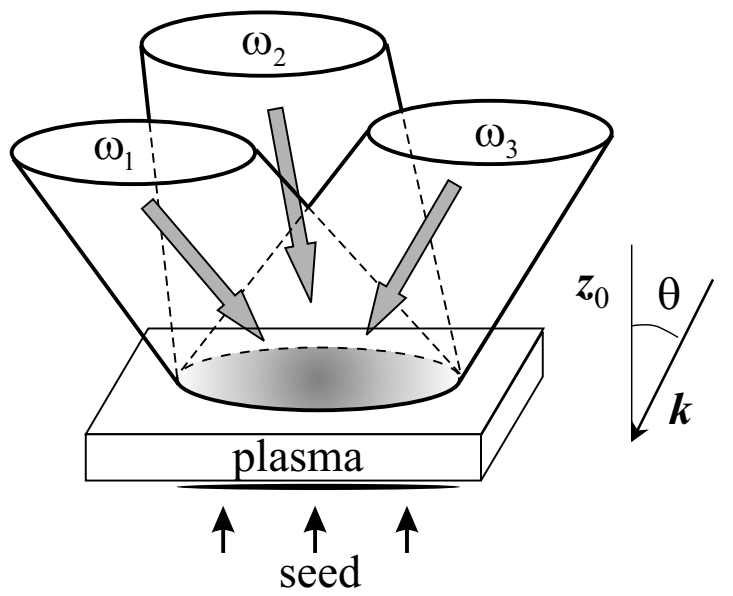

FIG. 1: Schematic of pump incident on plasma. 


$$
a=\frac{a_{0} F(r)}{\sqrt{N}} \sum_{n=1}^{N} \exp \left(i \Delta \boldsymbol{k}_{n} \cdot \boldsymbol{r}-i \Delta \omega_{n} t+i \phi_{n}\right),
$$

where $\boldsymbol{k}_{0}=\boldsymbol{z}^{0} \omega_{0} / c$.

As seen from Fig. 2, a mono-frequency pump (of sufficiently small angular spread $\theta$ in order few degrees) produces long speckles which spoils the output pumped pulse focusability. A relatively small frequency spread (less or order of the linear growth rate $\gamma$ ) in multi-frequency pump bends and breaks the speckles.
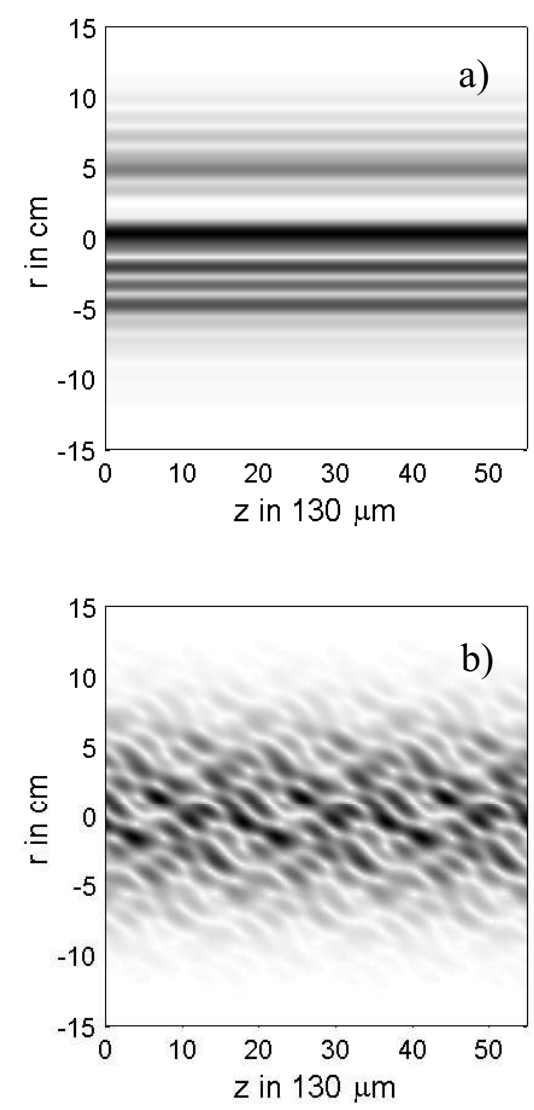

FIG. 2: An example of the spatial intensity patterns for monofrequency (a) and multi-frequency (b) pumps consisting of 7 sub-beams; darker region correspond larger pump intensity.

We expect a twofold effect of such a "mixed" pump. First, the interference of different beams constituting the pump wave enhances the amplified pulse focusability, primarily spoiled by speckled structure of the pump: On each geometric ray of the amplified pulse, the amplification gain is determined by the local (in the transverse direction) pump intensity. If the latter varies significantly across the interaction region, during amplification, the transverse seed structure evolves into multiple, extremely intense peaks. Because of their small spatial scale, each of those diffracts drastically, and thus the whole pulse cannot be focused efficiently into a narrow spot after amplification.

To demonstrate this effect in more detail, one can represent the focused pulse amplitude $b_{\text {foc }}$ in terms of the transverse Fourier spectrum of the pumped pulse after plasma, $\left(b_{\mathrm{amp}}\right)_{\boldsymbol{k}_{\perp}}$. As can be shown easily for $2 \mathrm{D}$ case,

$$
b_{\mathrm{foc}}\left(\boldsymbol{r}_{\perp}\right)=2 \sqrt{\pi \alpha}\left(b_{\mathrm{amp}}\right)_{\boldsymbol{k}_{\perp}=2 \alpha \boldsymbol{r}_{\perp}},
$$

where $\alpha=k_{0} / F$ is the phase front curvature of the amplified pulse, and $F$ stands for the focal length. From Eq. (6), it follows that the spatial width of the focused pulse is proportional to the spectral width of the pulse before focusing. Thus, narrow peaks in the latter would result in focal spot broadening, i. e. bad focusability of the amplified pulse. Using a pump wave consisting of multiple beams prevents speckles formation because of inter-beam phase mixing, which effectively averages out the speckled structure during amplification and thus leads to better focusability. At the same time, the inhomogeneity of the pump phase front itself is "absorbed" by the plasma wave (see Ref. [11]), and thus does not impact the phase front of the output radiation.

The second effect produced by multiple pump beams of different frequencies consists of decreasing the amplification gain of plasma thermal fluctuations and seed precursors (considered as a sort of electromagnetic noise). Since each spectral component of plasma noise can be amplified at most by one of the beams, the linear increment of thermal fluctuations exponential growth $\gamma$ is effectively reduced by a factor of $\sqrt{N}$, since $\gamma$ is linear with respect to the amplitude of the resonant pump. On the other hand, the nonlinear amplification of the desired signal is determined by its interaction with the whole pump, because of the seed pulse spectrum broadening provided by the nonlinear compression of the amplified pulse. Furthermore, additional chirping of each of the beams is numerically shown to stabilize Raman instability of thermal fluctuations and precursors, and, what is most important, using pump wave formed by multiple beams of different frequencies allows suppressing the parametric instability appearing at $l_{\text {corr }} \gamma / c=1$ (see above) [7].

\section{FOCUSABILITY OF THE PUMPED PULSE}

Consider more specifically the effect of speckles on focusability of the pumped output pulse in a plasma without thermal or quasi-static density fluctuations. Assume also seed precursors to be absent. Fig. 3a shows the relative focused pulse intensity $\eta$ which is a fraction of the focused pulse maximal intensity that survives in real pump (compared to ideal non-speckled pump) as a function of the number of beams $N$ constituting the pump. Here $\Delta \omega$ is the spectral half-width of the pump. For a monofrequency pump $(\Delta \omega=0$, dashed line), the focused intensity is extremely poor and constitutes less than $30 \%$ of $I_{\max }$. However, even a small pump spectrum broadening $(\Delta \omega=0.1 \gamma)$ leads to substantial focusability enhancement. Spectrum broadening with $\Delta \omega=\gamma$ (solid line) almost completely restores the focusability.

Note that an excessive increase of the pump bandwidth reduces the fraction of pump energy transferred to the 

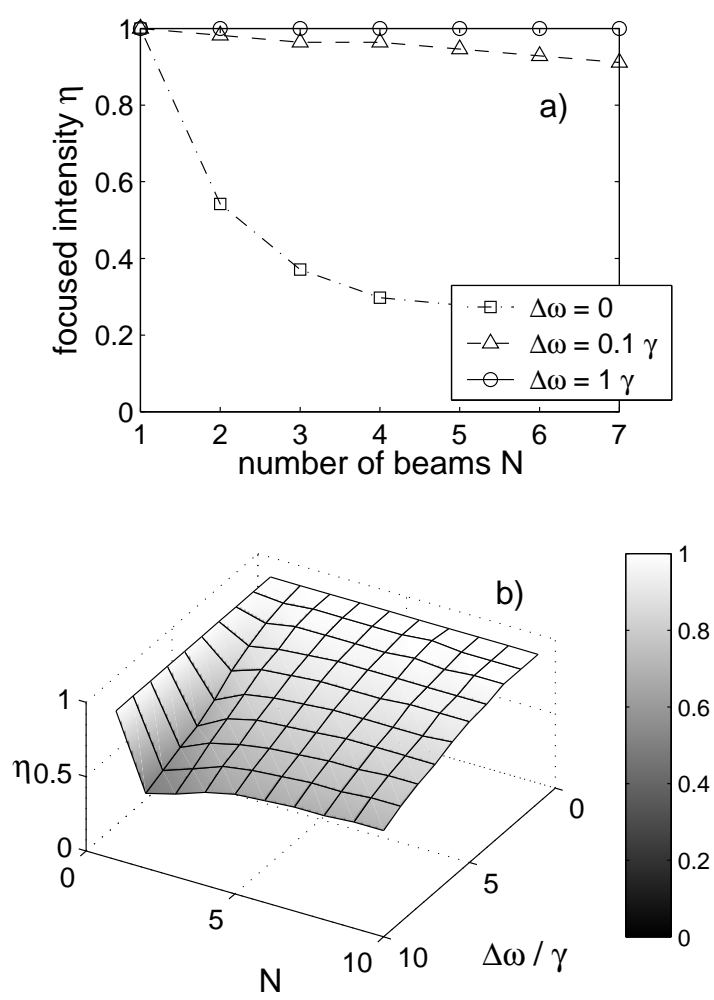

FIG. 3: The relative focused pulse intensity $\eta$ versus the number of the beams $N$ constituting the pump; $\Delta \omega$ stands for the spectral half-width of the pump.

pumped pulse because of the detuning out of resonance, which effect can be seen in (Fig. 3b), especially for a pump of 2 beams. The numerical simulation shows that the efficiency of amplification and, as result, the focused intensity, decreases with increasing spectral width. However, the effect diminish for a pump containing many beams, since a strong enough seed might absorb energy from several beams in an early stage of amplification.

As example of focusability enhancement the comparison of focused seed pulses for cases of beams with equal frequencies and with different frequencies is presented in Fig. 4. The darker regions correspond to larger seed amplitude. Note that the use of pump beams with multiple frequencies results in a larger and more highly focused output, which is ever more apparent at the focus plane than it is at plasma exit.

There is an interesting advantage of thermal noise in the plasma. Since the thermal noise depletes the pump preferably when the pump is largest, the pump profile encountring the pulse will tend to be transversely flat. The seed amplified by this pump tends to be easer to focus.

For example on the Fig. 5 we show the comparison between amplification and focusing in the ideal case (without thermal or density fluctuation) and in the presence of thermal fluctuation. The amplitude of the thermal fluctuations corresponds to a temperature $40 \mathrm{eV}$. It is easy to
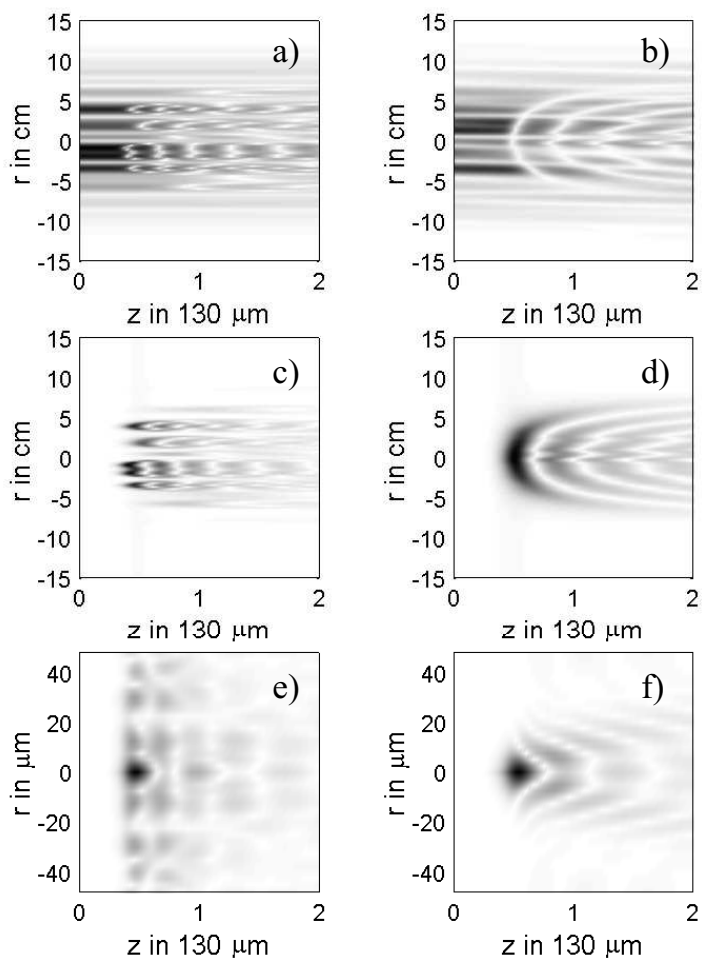

FIG. 4: An example of the spatial distribution of pump amplitude ( $\mathrm{a}$ and $\mathrm{b})$, seed amplitude at the plasma exit ( $\mathrm{c}$ and $\mathrm{d}$ ) and at the focus plane (e and f) for pump beams with equal frequency ( $\mathrm{a}, \mathrm{c}$ and e) and for pump beams with different frequencies ( $b$, $d$ and $\mathrm{f}$ ). Pump contains 7 beams. Darker regions correspond to larger pump intensity.
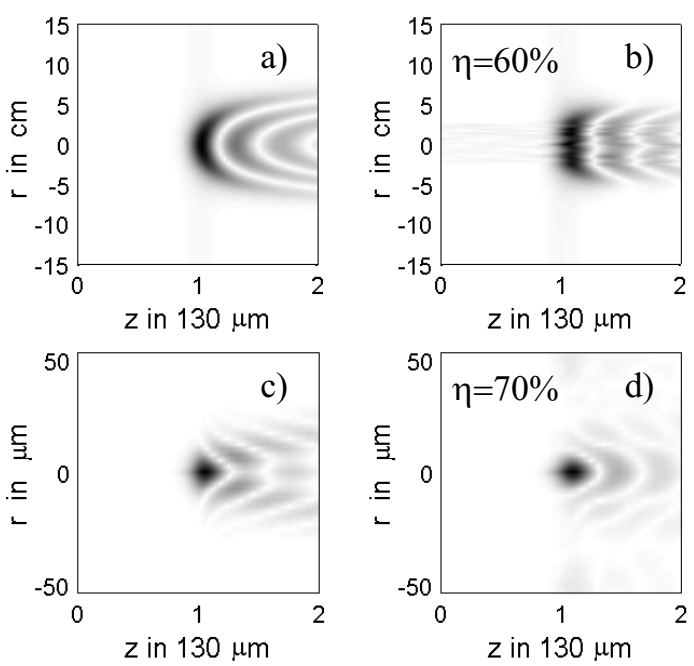

FIG. 5: An example of the spatial distribution of seed amplitude at the plasma exit ( $\mathrm{a}$ and $\mathrm{b}$ ) and at focus plane (c and d) for the ideal situation (without thermal or density fluctuations, a and c) and for plasma with noise and density fluctuations (b and d). Darker region correspond larger pump intensity. 
see that, in spite of sufficient losses during amplification (intensity of seed nearly twice smaller), the remaining energy can be better focused. In this case the seed retains an intensity at focus only on one third smaller than in the ideal case.

\section{NOISE GROWTH SUPPRESSION}

Consider now how the multiple beam pump structure influences the noise amplification. As pointed out above, one of the most challenging problems for the realization of the plasma Raman amplifier consists of suppressing the amplification of plasma thermal fluctuations and seed precursors. Though, in uniform plasmas, this problem can be solved by linearly changing the pump frequency [2]. The presence of plasma inhomogeneities can lead to another parametric instability, completely undermining the stabilizing effect of pump chirping. However the numerical simulations given below show that using pump wave formed by multiple beams of different frequencies can suppress this instability. Also the original Raman instability of plasma thermal fluctuations (and seed precursors considered as a sort of electromagnetic noise) is slowed down by a factor of $\sqrt{N}$. Furthermore, chirping each of the beams constituting the pump wave leads to complete stabilization of the noise growth.

Note that one needs the pump badwidth to be as wide as possible in order to avoide backscatter from noise in the linear regime of amplification. On the other hand, the bandwidth should be small enough so that the amplification of the useful seed, which is already in a nonlinear regime, is not disturbed. There is a window of opportunity to accomplish this, because the useful bandwidth of the pump in the nonlinear stage is increased with increasing seed intensity. However, for linear stage (for thermal noise) the amplifiable bandwidth is small enough (on the order of $\gamma$ ).

We found numerically that, for plasma length of 55 increments, the optimal bandwidth for efficient BRA is of order of $\Delta \omega=2 * 10 \gamma$. Moreover we note that for a small number of beams (less than $7 \ldots 10$ ), the dominant parameter is the frequency difference between each two beams. For this case, the efficiency decreases with decreasing the number of beams. For the opposite case of large number of beams (more than 10), the dominant parameter is the maximum bandwidth of the full pump frequency spectrum for which the seed is still well amplified. For a large number of beams, the efficiency of BRA will not depend on number of beams. The examples simulated employ a "critical" number of beams (equal to 7 , which is essentially a large number) and for small number of beams (equal to 3 ).

The numerical simulations given below demonstrate the benefits of using a "mixed" pump in plasmas with thermal fluctuations and precursors. Calculations were performed for plasma with temperature of $40 \mathrm{eV}$ for pump containing $N=7$ beams with frequencies equally
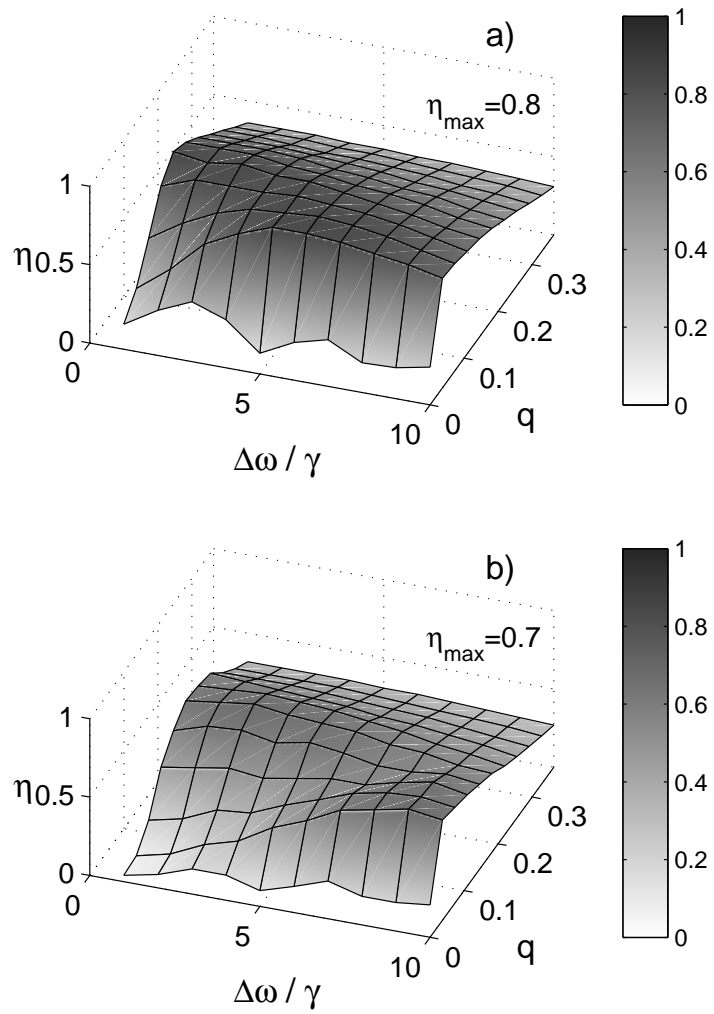

FIG. 6: The focused pulse relative intensity $\eta$ versus the parameter of pump chirping $q$ and the pump spectral width $\Delta \omega$. The plasma thermal fluctuations are calculated for electron temperature $40 \mathrm{eV}$; no quasi-static density perturbations are present $(\delta n / n=0)$ : upper figure - no precursors, lower figure - precursors included.

distributed over the interval $(-\Delta \omega, \Delta \omega)$ with random phases $\phi_{n}$ and equal chirping:

$$
a=\frac{a_{0} e^{i q \gamma^{2} t^{2} / 2} F(r)}{\sqrt{N}} \sum_{n=1}^{N} \exp \left(i \Delta \boldsymbol{k}_{n} \cdot \boldsymbol{r}-i \Delta \omega_{n} t+i \phi_{n}\right),
$$

where $q$ is the conventional parameter that determines the chirping rate.

The results for homogeneous plasma are given in Fig. 6. As stated above, in this case, chirped pump provides better noise suppression in comparison with a pump formed by multiple laser beams of different frequencies. The optimal value of the parameter of pump chirping $q$ coincides with the one predicted in Refs. [2, 3]. Including small precursors into the model (with the total energy equal to $10 \%$ of the original seed energy) does not influence significantly the amplification and the compression of the desired signal as can be seen from Fig. $6 \mathrm{~b}$. Numerical simulations show that about $70 \%$ of the total pump energy can be transferred to the focusable part of the amplified seed.

The addition of small random density perturbations $\delta n / n=1 \%$ decreases the efficiency of the chirped pump 

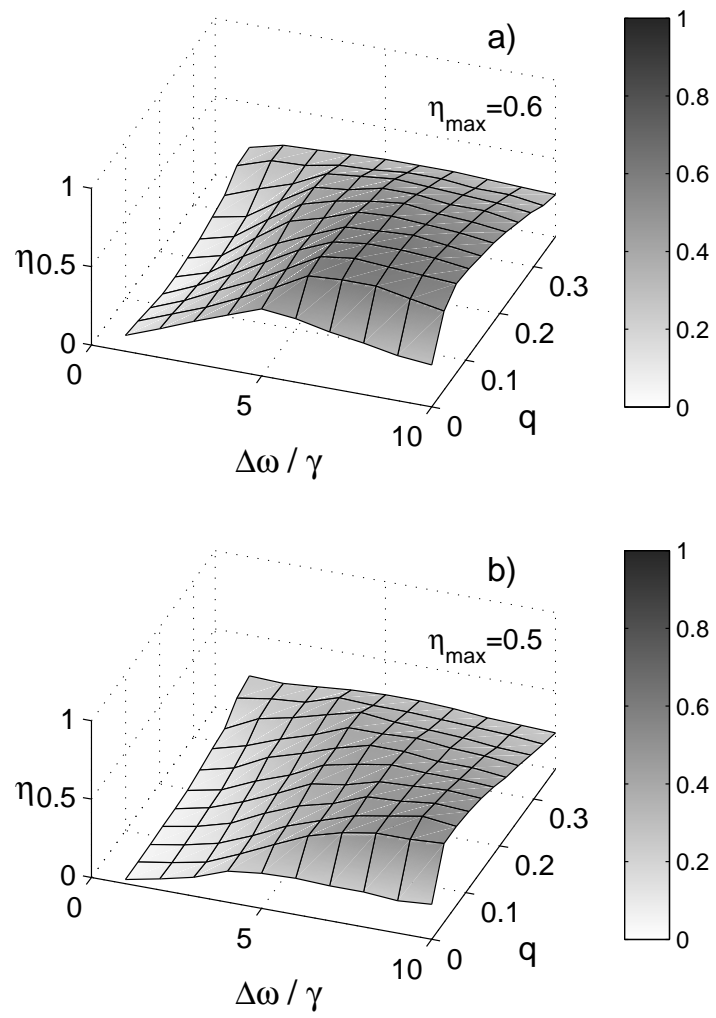

FIG. 7: The focused pulse relative intensity $\eta$ versus the parameter of pump chirping $q$ and the pump spectral width $\Delta \omega$. The plasma thermal fluctuations are calculated for electron temperature $40 \mathrm{eV}$; quasi-static density perturbations are present with $\delta n / n=1 \%$ and the correlation length $l_{\text {corr }}=300 \mu \mathrm{m}$ : upper figure - no precursors, lower figure precursors included.

scheme dramatically. Even for large-scale perturbations with $l_{\text {corr }}=300 \mu \mathrm{m}$ (Fig. 7 ), significant drop of the focused pump intensity can be seen in the case when the conventional chirped single-beam pump is used. Namely, less than $20 \%$ of the total pump energy is transferred to the focusable part of the amplified pulse. However, the multiple-beams scheme with $N=7$ results in about $50 \%$ of the maximal energy in focus, both with and without precursors. The optimal conditions, which readily follow from the results given in Fig. 7 are the following: $\Delta \omega=7-8 \gamma$ which corresponds to a $2 \gamma$ frequency difference between each two beam frequencies. The optimal values of chirping parameter $q$ has range $q \approx 0.05 \ldots 0.1$. Note that the optimal value of $q$ for the multiple-beam pump is $3 \ldots 4$ times smaller than the one for the conventional single-beam chirped pump in homogeneous plasma.

The similar picture can be seen for plasma with smaller correlation length of quasi-static density perturbations. But the efficiency of chirped pump decreases greater. It can transfer less than $5 \%$ of pump energy to the focused part of seed. However, the multiple-beams scheme with
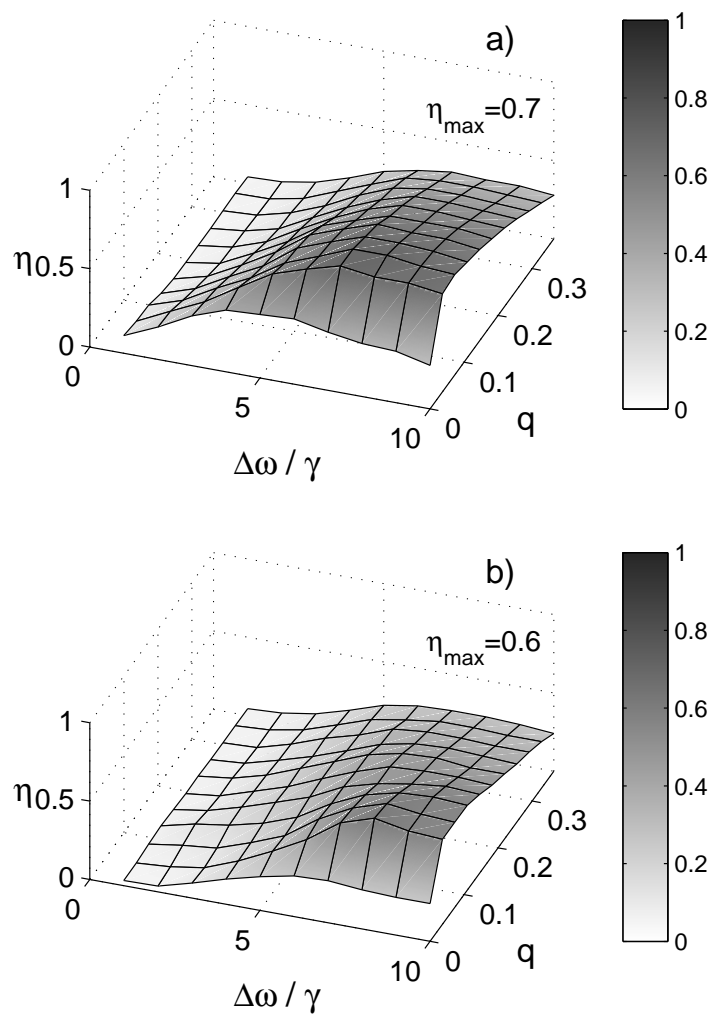

FIG. 8: The focused pulse relative intensity $\eta$ versus the parameter of pump chirping $q$ and the pump spectral width $\Delta \omega$. The plasma thermal fluctuations are calculated for electron temperature $40 \mathrm{eV}$; quasi-static density perturbations are present with $\delta n / n=1 \%$ and the correlation length $l_{\text {corr }}=130 \mu \mathrm{m}$ : upper figure - no precursors, lower figure precursors included.

$N=7$ still results in about $50 \%$ of the maximal energy in focus, both with and without precursors. The optimal conditions also stay the same as previously. The results of numerical simulations with $l_{\text {corr }}=130 \mu \mathrm{m}$ are presented in Fig. 8. We can even small increasing of focused intensity $\eta$ in comparison with case shown on Fig. 7. It takes place due to the next. The small density modulation has similar effect as some detuning due to pump chirping and help slightly to suppress the noises when the parametric instability absent.

Decreasing the number of beams is not recommended, since increasing the intensity of each beam leads to a higher growth rate of noise. This fact is demonstrated in Fig. 9. But even here, the amplification efficiency reach $40 \%$. This is twice higher than for a pump containing only one beam with chirping. The optimal parameters of the pump differ from the case of $N=7$. Better spectral width has value closed to $\Delta \omega \approx 3 \gamma \ldots 6 \gamma$ which corresponds to a $2 \gamma \ldots 4 \gamma$ frequency difference between each two beam frequencies. The optimal values of chirping parameter $q$ has range $q \approx 0.15 \ldots 0.25$ greater than in the case of 7 beams in pump. 

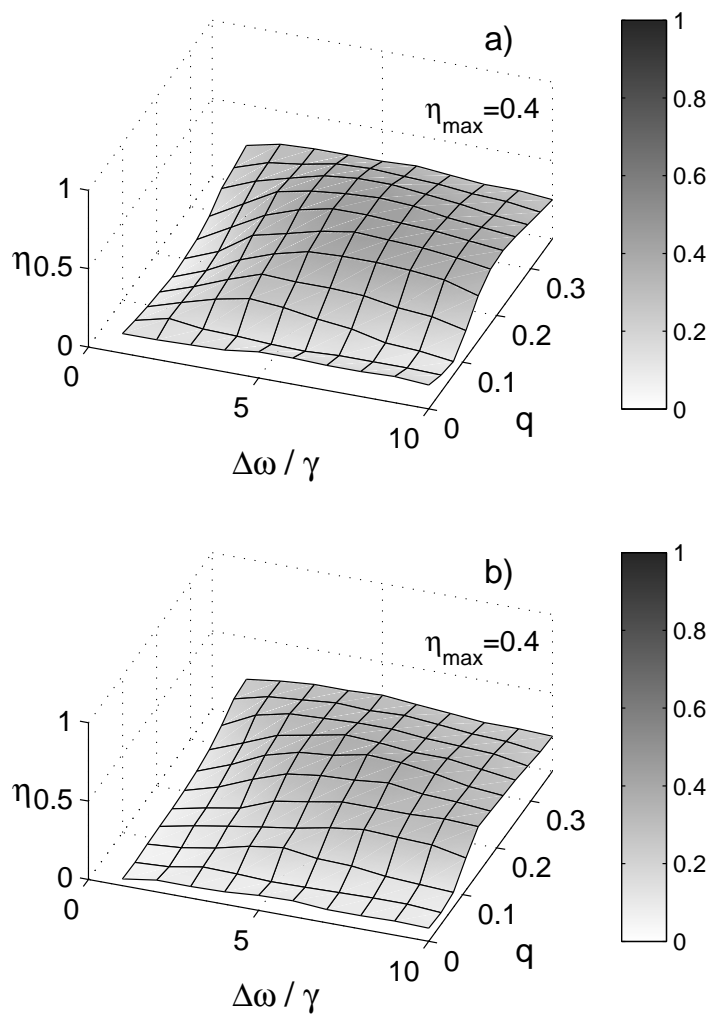

FIG. 9: The focused pulse relative intensity $\eta$ versus the parameter of pump chirping $q$ and the pump spectral width $\Delta \omega$ containing 3 beams. The plasma thermal fluctuations are calculated for electron temperature $40 \mathrm{eV}$; quasi-static density perturbations are present with $\delta n / n=1 \%$ and the correlation length $l_{\text {corr }}=300 \mu \mathrm{m}$ : upper figure - no precursors, lower figure - precursors included.

\section{SINGLE BEAM WITH COMPLEX CHIRP}

Similar effects could be achieved by a single-beam pump chirped in such a manner that its spectrum contains many frequencies, similar to the spectrum of pump containing several beams. For example the spectrum of the pump in the form

$$
a=a_{0} F(r) e^{i q \gamma^{2} t / 2+i \alpha \sin \omega_{s} t}
$$

can be easily found via Bessel functions. For example in figure 10 we present the spectrum for $\alpha=1.5$. It is easy to see that this spectrum has only 3 large enough harmonics and qualitatively similar to spectrum of pump containing 3 beams with constant frequency difference $\omega_{s}$ between each of them. As a consequence, we can expect that the pump in form (8) will have an effective noise suppression similar to pump containing 3 beams (Fig. 9 ). We made numerical simulation and obtain the similar figure (Fig. 11).

Increasing of the parameter $\alpha$ or the number of harmonics of the pump phase may lead to further increases

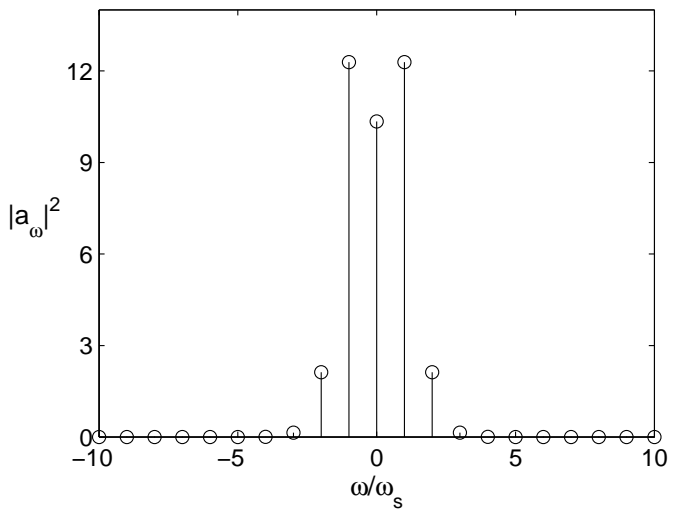

FIG. 10: The spectrum of pump (8) for $\alpha=1.5$ and $q=0$.

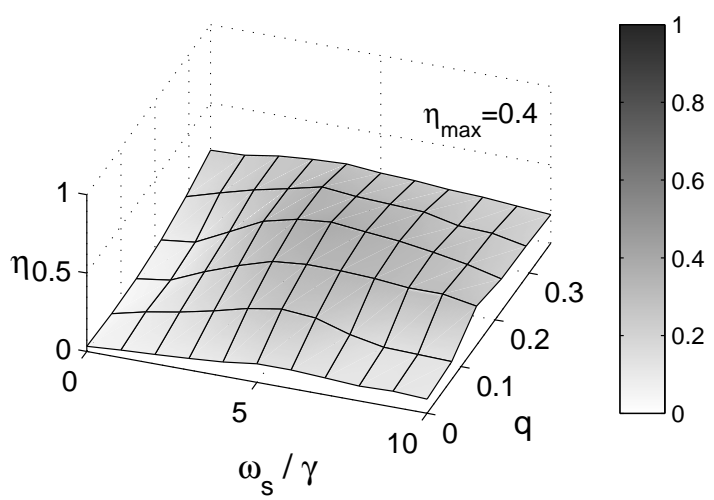

FIG. 11: The focused pulse relative intensity $\eta$ versus the parameter of pump chirping $q$ and the pump spectral width $\Delta \omega$ in form (8) for $\alpha=1.5$. The plasma thermal fluctuations are calculated for electron temperature $40 \mathrm{eV}$; quasi-static density perturbations are present with $\delta n / n=1 \%$ and the correlation length $l_{\text {corr }}=300 \mu \mathrm{m}$, precursors included.

of the relative intensity $\eta$. As in case of several beams we can achieve relative intensity at level of $60 \%$. At the same time for a wider pump spectrum (8) the picture become sensitive for variation of parameters ( $\alpha$ or frequencies of pump phase). This small variation can sufficiently change relative intensity $\eta$ up to $2 \ldots 3$ times. So devices which prepare necessary phase of single beam should be very precise. Increasing the parameter of linear chirping $q$ make the figures smoother but decreases the maximal relative intensity $\eta$ to level $40 \ldots 50 \%$ which is close to shown in Fig. 11. Decreasing the parameter $\alpha$ is not recommended due to pump spectrum become too tight and no longer stabilize the parametric instability.

For example on figure 12 we present result of numerical simulation for pump with phase containing 2 sinusoid with different frequencies and linear chirping:

$$
a=a_{0} F(r) \exp \left(i \alpha \sin \omega_{s 1} t+\alpha \sin \omega_{s 2} t+i q \gamma^{2} t / 2\right)
$$

As early we see practically absence of useful seed amplification for case than only linear chirping present with frequencies $\omega_{s}=0$. For low values of linear chirping $q$ we 


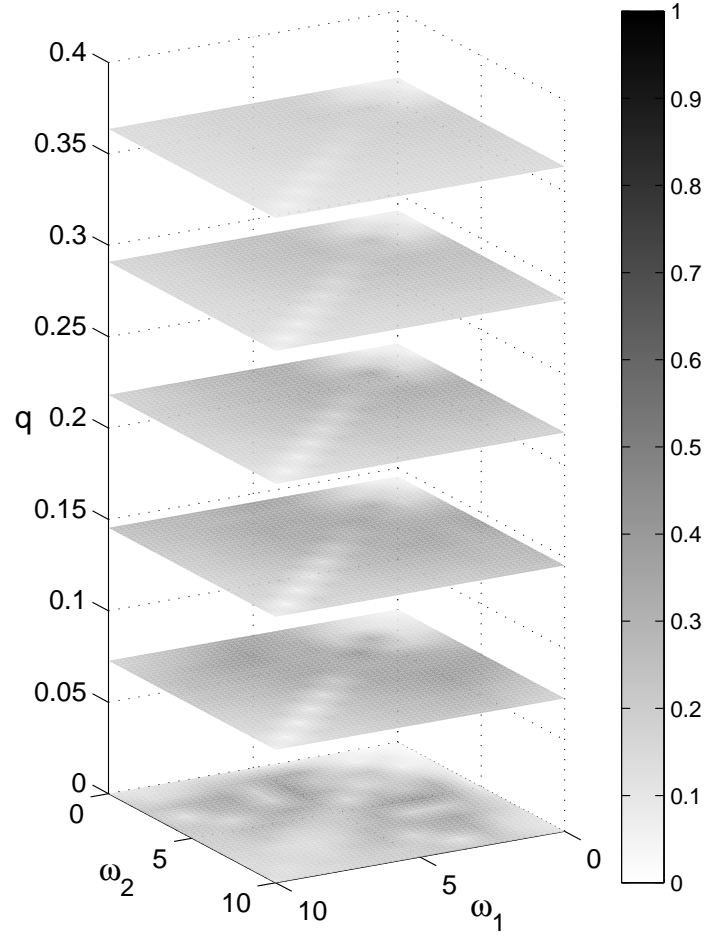

FIG. 12: The focused pulse relative intensity $\eta$ versus the parameter of pump chirping $q$ and the frequencies $\omega_{s}$ for pump in form (9) with $\alpha=0.5$. The plasma thermal fluctuations are calculated for electron temperature $40 \mathrm{eV}$; quasi-static density perturbations are present with $\delta n / n=1 \%$ and the correlation length $l_{\text {corr }}=130 \mu \mathrm{m}$, precursors included.

see rather complex distribution with narrow peaks. The value of focused intensity is sensitive for small variation of frequencies $\omega_{s}$ or parameter $q$. With increasing the linear chirping the distribution become smother but the maximum of focused intensity decreases. As result the focused intensity will have the values close to the case shown on figure 11, i. e. to level of $40 \%$ in optimal case.

The physical picture of process is also rather clean. Let consider pump (8) with defined parameter $\alpha=1.5$ and some small enough chirping $q$. First of all the strong sinus chirp of pump phase will prevent appearing of the parametric instability but still have dangerous places where the frequency of pump varies slow. These places lie in maximums of sinus (Fig. 13). In case of linear chirping absence an noise with frequency $\delta \omega \approx 4 \gamma$ can effectively amplified along all maximum. The exponential growth of these noises will weaker than in case of ideal pump but fast enough. As result the pump will be rather depleted. Appearance of small linear chirping will shift the cosine maximums in vertical direction so that noises in linear stage can growth only on limited number of maximums. So presence of linear chirping will limited the number of dangerous maximums for each noise wave by 3 for this example. Other maximums will have large enough detun-

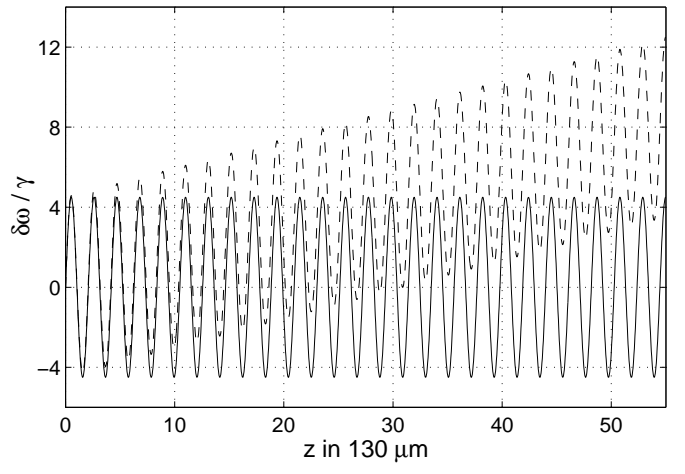

FIG. 13: The scheme of dangerous places on phase chirped with sinus with (dash line) and without (solid line) linear chirping. The sinus frequency is $\omega_{s}=3 \gamma$ and parameter $\alpha=$ 1.5

ing and will not influence to this noise wave. As result the growths of noises will be stabilized. Of course this stabilization will worse than just chirp stabilization in homogeneous plasma but in distinguish with linear chirp the combined (sinus and linear) still work in inhomogeneous plasma.

The only limitation of this scheme (scheme of combined chirping) is amplification of useful seed. High enough frequency of sinus chirping will prevent not only noise growth but also the seed amplification. So there are some window in parameters. Numerical simulation give us the optimal parameters of sinus frequency as $\omega_{s}=4 \ldots 6 \gamma$ for $\alpha=1.5$. The value of chirping $q$ has wider diapason $q \approx 0.15 \ldots 0.25$. This region of parameters provide amplification efficiency at level $40 \%$. These optimal parameters are closed to optimal parameters of pump containing 3 beams. This fact is direct consequence of written above similarity between MBP scheme and scheme of combined (linear and sinusoidal) chirping.

\section{DISCUSSION AND CONCLUSION}

A number of practical recommendations for experimental realization of the plasma Raman amplifier with multiple-beam pump flow from these results. First, consider those connected with pulse focusability. In uniform plasma, where the influence of thermal fluctuations and seed precursors instabilities is negligible, the focusability itself is relatively easy to provide. As seen from the numerical simulations presented above and those, obtained but not included into the paper, already two beams with frequency difference $\Delta \omega \approx 0.1 \gamma$ are enough to prevent the appearance of large-scale speckles in the pump structure and provide almost ideal focusability with efficiency $\eta>90 \%$.

On the other hand, suppressing the instability of plasma thermal fluctuations, which is the second reason for using multiple-beam pumps, represents a much more significant challenge. First of all, in order to deal with 
those, a large number of beams is found to be necessary in order to decrease the linear increment of Raman instability by distributing the pump energy over a larger bandwidth (which scales like $1 / \sqrt{N}$, as discussed above). On the other hand, distributing the pump energy over too wide a bandwidth will result in poor absorption of the pump by the desired signal, which would lead to inefficient amplification of the latter. The optimum for constraining the amplification of thermal fluctuations, while sustaining decent amplification gain of the desired signal, was numerically found to correspond to $N=7-10$ beams, distributed over the spectral width $\Delta \omega \sim N \gamma$. However, if one uses a sufficiently intense seed, which enters the nonlinear amplification stage from the very beginning of the interaction process, the number of the beams can be made larger and the pump bandwidth can be increased proportionally. In this case, because of a broader spectral width of the desired signal, amplification by a larger number of pump beams can be sustained, while the growth of plasma thermal fluctuations will be slowed down inverse proportionally to a larger $\sqrt{N}$.

Another parameter, which can also be optimized in order to achieve better results for Raman amplification of the desired signal, is the pump frequency chirping $q$. Two major limitations are imposed. First of all, $q$ cannot be too large, since, at larger $q$, the pump propagates through the seed pulse without substantial absorption, and the efficiency of the desired signal amplification decreases significantly. On the other hand, sufficiently large chirping is needed in order to stabilize the instability of the plasma thermal fluctuations together with parasitic amplification of seed precursors. With these limitation taken into account, the optimal value of the parameter of chirping found numerically is $q \lesssim 0.1$, which, though it varies slightly depending on plasma parameters, is significantly smaller the one predicted for homogeneous plasma $[2,3]$.

Summarizing, we suggest a novel concept of a so-called "mixed" pump for the promising scheme of short ultraintense laser pulses amplification by Raman backscattering in plasmas. Mixed pump represents a superposition of several laser beams of slightly different frequencies and allows a number of advantageous properties of the desired signal amplification. Among those, using a mixed pump can result in decreasing the growth rate of the instabilities of plasma thermal fluctuations and seed precursors, while simultaneously providing enhanced focusability of the desired signal. Combining the multiple beam scheme with small chirping of each beam has a cumulative effect that effectively suppresses the noise amplification in nonuniform plasmas where using the conventional singlebeam pump is not efficient. Even with thermal fluctuations and seed precursors, a mixed pump can result in the transfer up to $50 \%$ of the pump wave total energy into the well-focusable part of the amplified pulse.

We acknowledge valuable discussion with I.Y. Dodin and A.A. Solodov.

The work was supported by RFBR grants No 02-0217275, 02-02-17277, US DOE under contract DE-AC0276 CHO3073 and the US DARPA.
[1] N. J. Fisch and V. Malkin, Generation of Ultra-high Intensity Laser Pulses, Physics of Plasma 9, 2056-2063 (May, 2003).

[2] V. M. Malkin, G. Shvets, and N. J. Fisch, Phys. Plasmas, 7, 2232 (2000); V. M. Malkin, G. Shvets, and N. J. Fisch, Phys. Rev. Lett. 82, 4448 (2000).

[3] V. M. Malkin, G. Shvets, and N. J. Fisch, Phys. Rev. Lett. 84, 1208 (2000).

[4] Yu.A. Tsidulko, V. M. Malkin, and N. J. Fisch, Phys. Rev. Lett. 88, 236004 (2002).

[5] J.J. Thomson, Nuc. Fusion. 15, 237 (1975).

[6] A. Solodov, V. M. Malkin, and N. J. Fisch, Random density inhomogeneities and focusability of the output pulses for plasma-based powerful backward Raman amplifiers,
Physics of Plasma 10, 2540-2544 (June, 2003).

[7] D.R. Nicholson and A.N. Kaufman, Phys. Rev. Lett. 33, 1207 (1974); D.R. Nicholson, Phys. Fluids, 19, 889 (1976).

[8] I. Y. Dodin, G. M. Fraiman, V. M. Malkin, and N. J. Fisch, Sov. JETP 95, 625 (2002) [Zh. Eks. Teor. Fiz. 122, 723 (2002)].

[9] G.M. Fraiman, E.M. Sher, A.D. Yunakovsky, W.Laedke, Physica D., V.87, pp. 325-334 (1995).

[10] R.L. Berger,C.H. Still, E.A. Wiliams and A.B. Langdon, Phys. Plasmas 5, 4337 (1998).

[11] G. M. Fraiman, N. A. Yampolsky, V. M. Malkin, and N. J. Fisch, Phys. Plasmas, 9, 3617 (2002). 


\section{External Distribution}

Plasma Research Laboratory, Australian National University, Australia

Professor I.R. Jones, Flinders University, Australia

Professor João Canalle, Instituto de Fisica DEQ/IF - UERJ, Brazil

Mr. Gerson O. Ludwig, Instituto Nacional de Pesquisas, Brazil

Dr. P.H. Sakanaka, Instituto Fisica, Brazil

The Librarian, Culham Laboratory, England

Mrs. S.A. Hutchinson, JET Library, England

Professor M.N. Bussac, Ecole Polytechnique, France

Librarian, Max-Planck-Institut für Plasmaphysik, Germany

Jolan Moldvai, Reports Library, MTA KFKI-ATKI, Hungary

Dr. P. Kaw, Institute for Plasma Research, India

Ms. P.J. Pathak, Librarian, Insitute for Plasma Research, India

Ms. Clelia De Palo, Associazione EURATOM-ENEA, Italy

Dr. G. Grosso, Instituto di Fisica del Plasma, Italy

Librarian, Naka Fusion Research Establishment, JAERI, Japan

Library, Plasma Physics Laboratory, Kyoto University, Japan

Research Information Center, National Institute for Fusion Science, Japan

Dr. O. Mitarai, Kyushu Tokai University, Japan

Dr. Jiangang Li, Institute of Plasma Physics, Chinese Academy of Sciences, People's Republic of China

Professor Yuping Huo, School of Physical Science and Technology, People's Republic of China

Library, Academia Sinica, Institute of Plasma Physics, People's Republic of China

Librarian, Institute of Physics, Chinese Academy of Sciences, People's Republic of China

Dr. S. Mirnov, TRINITI, Troitsk, Russian Federation, Russia

Dr. V.S. Strelkov, Kurchatov Institute, Russian Federation, Russia

Professor Peter Lukac, Katedra Fyziky Plazmy MFF UK, Mlynska dolina F-2, Komenskeho Univerzita, SK-842 15 Bratislava, Slovakia

Dr. G.S. Lee, Korea Basic Science Institute, South Korea

Institute for Plasma Research, University of Maryland, USA

Librarian, Fusion Energy Division, Oak Ridge National Laboratory, USA

Librarian, Institute of Fusion Studies, University of Texas, USA

Librarian, Magnetic Fusion Program, Lawrence Livermore National Laboratory, USA

Library, General Atomics, USA

Plasma Physics Group, Fusion Energy Research Program, University of California at San Diego, USA

Plasma Physics Library, Columbia University, USA

Alkesh Punjabi, Center for Fusion Research and Training, Hampton University, USA

Dr. W.M. Stacey, Fusion Research Center, Georgia Institute of Technology, USA

Dr. John Willis, U.S. Department of Energy, Office of Fusion Energy Sciences, USA

Mr. Paul H. Wright, Indianapolis, Indiana, USA 
The Princeton Plasma Physics Laboratory is operated by Princeton University under contract with the U.S. Department of Energy.

\author{
Information Services \\ Princeton Plasma Physics Laboratory \\ P.O. Box 451 \\ Princeton, NJ 08543
}

Phone: 609-243-2750

Fax: 609-243-2751

e-mail: pppl_info@pppl.gov

Internet Address: http://www.pppl.gov 